\title{
Nuntia ad Floram Japoniae XVI.
}

\author{
auctore
}

\section{Honda}

Received February 10, 1932.

126) Senisetum Honda gen. nov. (Poaceae)

Herba perennis, caespitosa, foliis angustis. Panicula terminalis, ramosissima, ramulis filiformibus. Spiculae parvae, 1-florae, rhachilla supra glumas vacuas articulata, ultra florem non producta, flore hermaphrodito. Glumae 3; 2 inferiores vacuae, sub articulatione persistentes, carinatae, acuminatae, exaristatae; tertia florens brevior, tenuiter hyalina, dorso ad basin longe aristata, apice 1-2 aristata et nervis lateralibus 4 in setas tenues florem excedentes excurrentibus instructa. Palea obsoleta. Pili calli brevissimi persparsi. Stamina 3. Stylus brevissimus, stigmatibus 2.-Species unica.

Senisetum Hideoi (OHwI) Honda comb. nov.

Agrostis Hideoi OHwI in Tokyo Bot. Mag. XLIV. (1930) p. 568;

Makino et Nemoto Fl. Jap. ed. 2. (1931) p. 1304.

Nom. Jap. Okuyama-nukabo (nov.)

Hab.

Hondo: in monte Sasagamine, prov. Echigo (J. OHwI, anno 1929-typus in Herb. Imp. Univ. Tokyo); in monte Yukikuradake, prov. Echigo (ex J. OHwI); Otari, prov. Shinano (ex J. OHwI)

Planta endemica.

127) Achyranthes iaponica NAKAI

var. hachijoensis HoNda var. nov.

Folia supra glabra v. glabriuscula, nitidula, subtus praeter nervos puberulos glabrescentia.

Nom. Jap. Hachijō-inokozuchi (nov.)

Hab.

Hondo: Mitsune, ins. Hachijō (M. OGata, anno 1921-typus in Herb. Imp. Univ. Tokyo); ins. Aogashima (N. Matsuzaki, anno 1920).

Liukiu: ins. Okinawa (T. Mryagi); ins. Yonakuni (Y. Shimada, anno 1917).

128) Acer mono Maximowicz

var. dissectum (Wesmael) Honda nom. nov. 
Acer pictum var. dissectum Wesmael in Bull. Soc. bot. Belgique XXIX. (1890) p. 56; PAX in ENGLer Pflanzenreich 8. Heft (IV. 163). (1902) p. 47; Kordzum in Journ. Coll. Sci. Imp. Univ. Tokyo XXXII. (1911) p. 63 pro parte, fig. 9 a ; Matsumura Ind. Pl. Jap. II. (1912) p. 331; Makino et Nemoto Fl. Jap. (1925) p. 602, et ed. 2. (1931) p. 697.

Accr pictum var. angustilobum Makino in Tokyo Bot. Mag. VI.(1892) p. (51) nom. tantum.

Nom. Jap. Asahi-kaede.

Enkō-kaede.

Hab.

Hondo: Aone, prov. Rikuzen (S. Kusano, anno 1897); Nikkō; prov. Shimotsuke (S. HAtToRI, anno 1922); in monte Ōyama, prov. Sagami (S. Matsuda, anno 1900); Hakone, prov. Sagami (J. Matsumura, anno 1881); ibidem (G. Kordzumi, anno 1910); in monte Amagi, prov. Izu (S. Kodama); ibidem (T. NakaI, anno 1919); Yugashima, prov. Izu (J. Matsumura, anno 1883); Kawazu, prov. Izu (J. Matsumura, anno 1883).

Shikoku: in monte Tsurugi, prov. Awa (J. NikaI, no. 1302, anno 1904); ibidem (J. NikaI, no. 1458, anno 1905).

Planta endemica.

var. subtrifidum (MAKINO) HoNDA nom. nov.

Acer pictum var. dissectum subvar. subtrifidum Makino in Tokyo Bot. Mag. XVIII. (1904) p. 114; Kordzumi l. c. p. 64; Matsumura 1. c.; Makino et Nemoto l. c.

Nom. Jap. Yaguruma-kaede (T. Makino)

Maruba-tōkaede.

Hab.

Hondo: Agano, prov. Musashi (Y. Satake, anno 1929); in monte Takao, prov. Musashi (Y. Kobayashi)

Planta endemica.

var. nikkoense Hords var. nov.

Acer pictum var. dissectum (non Wesmael) Koidzumi l. c. p. 63 pro parte.

Folia palmato 5-7-partita, laciniis anguste oblongis acuminatis, subtus secus nervos pubescentia.

Nom. Jap. Urage-enkōkaede (nov.)

Hab.

Hondo: Fukushima, prov. Iwashiro (G. NAkahara, anno 1904); Nikkō, prov. Shimotsuke (S. Komatsu-typus in Herb. Imp. Univ. Tokyo); ibidern (T. Uchryama, anno 1901); ibidem (Y. Yамамото, anno 
1923); ibidem (M. Honda, anno 1924); ibidem (S. NAKAJIMA); inter montes Mitake et Otake, prov. Musashi (M, Furukawa); Abe, prov. Suruga (J. Sugrmoto, no. B 2, anno 1922).

Planta endemica. var. glaucum (KoIDzUMI) Honda nom. nov.

Acer pictum var. glaucum Kordzumi l. c. p. 64; Makino et Neмотo l. c. Nom. Jap. Urajiro-itaya (G. KordzumI)

Hab.

Hondo: in monte Mogariyama, prov. Uzen (S. Ishizuka, no. 136)

129) Scirpus mucronatus LinNaEUs

var. tataranus Honda var. nov.

Spiculae numerosae, breves, ovatae. Culmi triquetri, angulis alatis, truncatis.

Nom. Jap. Tatara-kangarei (nov.)

Hab.

Hondo: palus Tatara, prov. Kōzuke (H. Sекгмото, no. 3, anno 1931typus in Herb. Imp. Univ. Tokyo)

Planta endemica.

130) Ambulia gratissima NAKAI

var. angustifolia HoNdA var. nov.

Folia lanceolata v. linearia, opposita v. verticillata.

Nom. Jap. Hosoba-shisokusa (nov.)

Hab.

Hondo: palus Tatara, prov. Kōzuke (H. Seкrмoтo, no. 2, anno 1931typus in Herb. Imp. Univ. Tokyo)

Planta endemica.

131) Ischaemum anthephroides $\mathrm{MIQUEL}_{\mathrm{Q}}$

var. subnudum HoNDA var. nov.

Folia utrinque pilis appressis basi plerumque tuberculatis vestita.

Spiculae tantum scabro-papillosae, non longe denseque villosae.

Nom. Jap. Hime-kekamonohashi (nov.)

Hab.

Hondo: Kugenuma, prov. Sagami (T. Momirama, no. 116, anno 1931typus in Herb. Imp. Univ. Tokyo)

Kiusiu: Ata, prov. Satsuma (Y. Dor, no. 144, anno 1928).

Planta endemica.

132) Anaphalis todaiensis Honda sp. nov.

Caulis erectus, circ. $30 \mathrm{~cm}$ altus, albo-lanatus, simplex, ad apicem

foliatus. Folia erecto-patula, lanceolata, apice acuta v. obtusa, margine 
integra, sessilia, utrinque albo-lanata, $3-4 \mathrm{~cm}$ longa, 5-8 mm lata. Inflorescentia corymbosa, densiuscula, circ. $10 \mathrm{~cm}$ lata, ramis numerosis, albo-lanatis. Capitula subglobosa, 3-4 mm lata. Bracteae involucri imbricatae, multi-seriatae; exteriores breves, $2.5 \mathrm{~mm}$ longae, ovatae, obtusae, scariosae, aráchnoideae; interiores oblongae, obtusae, $4 \mathrm{~mm}$ longae, supra medium albae, glabrae, infra medium scariosae leviter arachnoideae. Flores foeminei numerosi; corolla tubulosa, angusta, $3 \mathrm{~mm}$ longa. Pappi $3 \mathrm{~mm}$ longi, albi. Ovarium oblongum, 0.5-0.7 mm longum, glabrum.

Nom. Jap. Todai-hahako (nov.)

Hab.

Hondo: Todai, prov. Shinano (T. ŌTA, J. Kase, T. Senda, M. Furukawa, S. Matsunami et S. Mizutani, anno 1931-typus in Herb. Imp. Univ. Tokyo)

Planta endemica.

133) Leontopodium perniveum Honda sp. nov.

Caulis erectus, simplex, gracilis, circ. $20 \mathrm{~cm}$ altus, foliosus, araneosus. Folia sparsa, sessilia, lanceolata, acuta, 15-20 mm longa, 4-5 mm lata, supra cinereo-araneoso-tomentosa, subtus dense niveo-araneoso-lanata, nervis utrinque obscuris. Corymbus terminalis, dense aggregatus. Capitula subglobosa, dense albo-lanata. Squamae involucri lanceolatae, obtusae, circ. $3 \mathrm{~mm}$ longae, extus longe araneoso-albo-lanatae, intus in sicco brunneae, glabrae. Flos hermaphroditus tenuis, $1 \mathrm{~mm}$ longus, setis gracilibus, albis, scabris. Flos foemineus tenuissimus, $1.5 \mathrm{~mm}$ longus, ovario glabro.

Nom. Jap. Kawara-usuyukisō (nov.)

Hab.

Hondo: Todai, prov. Shinano (T. $\bar{O}_{\mathrm{TA}}$, J. Kase, T. Sknda, M. Furukawa, S. Matsunami et S. Mrzutani, anno 1931-typus in Herb. Imp. Univ. Tokyo)

Planta endemica.

134) Stachys baicalensis Fischer

var. angustifolia Honda var. nov.

Folia linearia v. lineari-lanceolata, $3-4.5 \mathrm{~cm}$ longa, $4-6 \mathrm{~mm}$ lata.

Nom. Jap. Hosoba-inugoma (nov.)

Hab.

Hondo: in pede montis Shirane, prov. Shimotsuke (M. Naramura, anno 1928-typus in Herb. Imp. Univ. Tokyo)

Planta endemica. 